\title{
MODELO DE ADEQUAÇÃO DA ESCÓRIA PARA TRATAMENTO DE PROTEÇÃO DO REVESTIMENTO REFRATÁRIO DO CONVERSOR*
}

\author{
Rafaela Pereira Batista ${ }^{1}$ \\ André Luiz Vasconcellos da Costa e Silva ${ }^{2}$ \\ José Adilson de Castro ${ }^{3}$ \\ Alan Alves Vieira ${ }^{4}$ \\ Antônio Augusto de Rezende Martins ${ }^{5}$ \\ Rafaela Pacheco Malvão dos Santos ${ }^{6}$ \\ Rodrigo da Silva Magalhães ${ }^{7}$
}

\section{Resumo}

O consumo de refratário na produção de aço é parte significativa no custo de produção. Por este motivo, é usual nas aciarias a oxigênio empregar os processos de slag splashing e slag coating, visando à formação de uma camada de escória sobre o refratário de $\mathrm{MgO}-\mathrm{C}$. Os dois procedimentos diferem, principalmente, pelo seu objetivo: o slag splashing visa a proteção do revestimento como um todo, enquanto o slag coating busca proteger principalmente o fundo do conversor e as ventaneiras do sopro combinado. Para a efetividade destes processos, é necessário adequar a escória para que a camada formada resista a temperatura e condições do processo, seja compatível quimicamente com o refratário e tenha boa aderência ao tijolo do forno. Este trabalho apresenta os resultados do desenvolvimento de um modelo matemático que orienta quanto a adição para o tratamento do revestimento refratário do conversor pela projeção de escória.

Palavras-chave: Conversor, Refratário, Slag Splashing,Slag Coating.

\section{MODEL FOR SLAG ADEQUACY FOR THE TREATMENT OF PROTECTION OF REFRACTORIES OF CONVERTER}

\section{Abstract}

Refractory consumption in steel production is a significant part of the cost of production. For this reason, it is usual for oxygen steelmaking to employ slag splashing and slag coating processes, aiming at the formation of a slag layer on the $\mathrm{MgO}-\mathrm{C}$ refractory. The two procedures differ mainly on their purpose: slag splashing aims at protecting the refractory lining as a whole, while slag coating seeks mainly to protect the bottom of the converter and the tuyeres of the combined blow system. For the effectiveness of these processes, it is necessary to adjust the slag so that the layer formed resists the process temperature and conditions, is chemically compatible with the refractory and has good adherence to the refractory brick. This work presents the results of the development of a mathematical model that defines the amount of material for the treatment of the refractory lining of the converter through slag projection.

Keywords: Converter, Refractory, Slag Splashing, Slag Coating.

1 Engenheiro Metalurgista, Engenheiro de Desenvolvimento Pl., Companhia Siderúrgica Nacional, Volta Redonda, RJ, Brasil.

2 Engenheiro Metalurgista, MASc, PhD, Professor, EEIMVR, Universidade Federal Fluminense, Volta Redonda,

3 Engenheiro Metalurgista, MSc, PhD, Professor, EEIMVR, Universidade Federal Fluminense, Volta Redonda, RJ, Brasil

4 Estudante, Engenharia Metalúrgia, Universidade Federal Fluminense, Volta Redonda, RJ, Brasil

5 Engenheiro Metalurgista, Coordenador de Projetos Especiais, Companhia Siderúrgica Nacional, Volta Redonda, RJ, Brasil.

6 Engenheiro Metalurgista,MSc, Engenheiro de Desenvolvimento Sr., Companhia Siderúrgica Nacional, Volta Redonda, RJ, Brasil

7 Engenheiro Metalurgista, MSC, Engenheiro Especialista, Companhia Siderúrgia Nacional, Volta Redonda, RJ, Brasil. 


\section{INTRODUÇÃO}

Em aciarias a oxigênio é comum realizar tratamentos, pós vazamento do aço, utilizando escória. Visando criar "camadas de sacrifício" sobre o tijolo refratário, a custo inferior a troca dos refratários e assim, prolongar a vida útil do revestimento do conversor. Tais procedimentos se baseiam na projeção e aderência de misturas de óxidos as paredes do conversor, criando um uma camada sob o revestimento. Para tal, é necessário (a) a produção, dentro do conversor, de misturas adequadas entre a escória do final de sopro e adições ricas em $\mathrm{CaO}$ e $\mathrm{MgO}$ e (b) o uso de técnicas de projeção que levem esta mistura a aderir as posições do conversor a serem protegidas. Dois procedimentos são os mais comuns: slag coating, que objetiva a proteção do refratário do fundo do conversor, empregando o basculamento parcial do conversor, e o slag splashing, que visa a proteção do revestimento como um todo projetando a mistura escória-adições com o uso de sopro de nitrogênio pela lança do conversor ${ }^{(1)}$.

Para que o processo seja eficaz é necessário que parte da mistura de óxidos formada consiga aderir aos refratários e que tenha composição química adequada para resistir a interação com a escória e/ou com o gusa dependendo da região em que for depositada. Além disto, é necessário que a mistura tenha propriedades físicas adequadas a sua projeção ou distribuição sobre o fundo do forno pelo basculamento. Assim, não é suficiente considerar apenas a composição química da mistura de óxidos e sua compatibilidade termodinâmica com o ambiente, mas também uma série de outras características dependentes da composição química. Uma das características importantes no processo de distribuição e aderência da mistura sobre os refratários é sua viscosidade. Camelli e colaboradores (2), entre outros, demonstraram a importância da viscosidade da escória nestes processos. A viscosidade aparente de uma mistura de óxidos depende da composição química do líquido, da temperatura (3) e da fração sólida (4). A importância da viscosidade sobre os aspectos físicos do splashing foi demonstrada, por exemplo, por Barron, Medina e Hilero ${ }^{(5)}$. Grosjean e Riboud ${ }^{(6)}$ concluíram, com base em estudos práticos, que uma escória com cerca de $20 \%$ de partículas sólidas proporcionaria a realização de um slag coating eficiente.

Outro método comumente empregado para avaliar a qualidade da escória em relação ao tratamento do revestimento refratário do conversor é a temperatura liquidus da escória. Quanto mais esta variável se distancia da temperatura de trabalho do conversor, mais resistente será a camada de sacrifício formada sob o refratário. Costa e Silva, Pereira e Chrisóstomo (10) desenvolveram, a partir de simulações por termodinâmica computacional, seguida por regressão multi-variada, uma equação capaz de estimar a temperatura liquidus da escória. A equação tem a forma da Equação 1 e é utilizada na rotina de operação dos conversores da CSN.

Temperatura Liquidus

Eq.1

$$
\begin{aligned}
& =a+(b x \% C a O)+(c x \% M g O)+\left(d x \% P_{2} O_{5}\right)-(e x \% M n O) \\
& -\left(f x \% A_{2} O_{3}\right)-(g x \% F e O)-\left(h x \% S_{0} O_{2}\right)
\end{aligned}
$$

Por outro lado, a compatibilidade química do revestimento formado pela projeção dependerá de quais fases sólidas estarão presentes na mistura entre escória e as adições de ajuste, após vazamento do aço, e da composição do líquido que promoverá sua adesão a parede.

As escórias dos conversores a oxigênio são compostas, majoritariamente, por composições no sistema $\mathrm{CaO}-\mathrm{SiO}_{2}-\mathrm{MgO}-\mathrm{FeO}$, ricas em $\mathrm{FeO}$ no início do sopro principalmente. A análise das fases sólidas presentes neste sistema é útil para a escolha das melhores composições. Avaliando-se os sistemas binários que compõe este sistema multi-componente, é possível verificar quais são as fases de alto ponto de fusão e potencial compatibilidade com escória e/ou gusa líquido. No sistema CaO- 
$\mathrm{SiO}_{2}$ (Figura 1A), além da $\mathrm{CaO}$ e do $\mathrm{SiO}_{2}$ existem duas fases com alto ponto de fusão: $\mathrm{C}_{3} \mathrm{~S}$ e $\mathrm{C}_{2} \mathrm{~S}$, com $2050^{\circ} \mathrm{C}$ e $2130^{\circ} \mathrm{C}$ respectivamente. Estas fases não têm, entretanto, boa resistência ao FeO, como mostra a Figura 1B: quanto maior o teor de $\mathrm{FeO}$ na escória, menor a temperatura liquidus da mistura. Assim, a introdução de aditivos ricos apenas em $\mathrm{CaO}$ não parece adequada para o tratamento de proteção do revestimento refratário no processo de produção de aço em conversor a oxigênio.

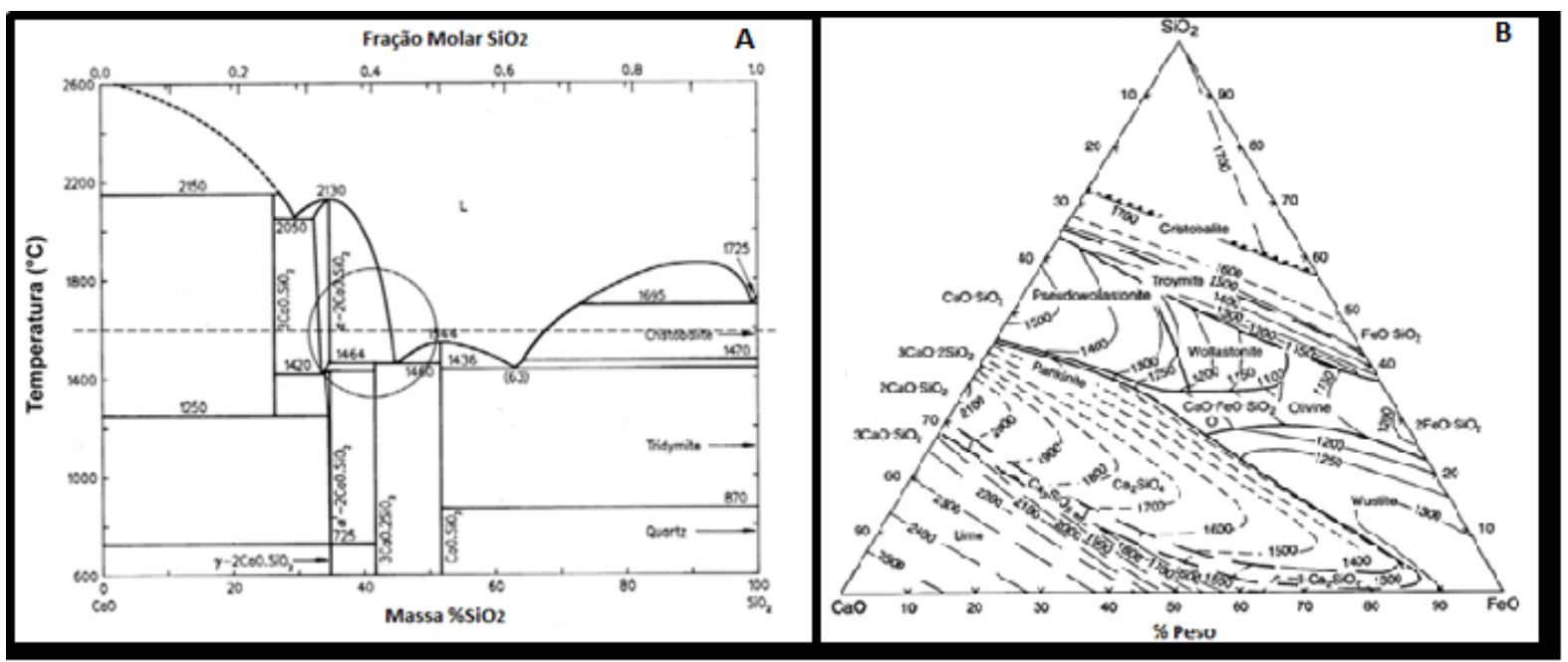

Figura 1 - A) Diagrama CaO-SiO2. B) Diagrama CaO-SiO2-FeO. Adaptados de (7).

No sistema $\mathrm{MgO}-\mathrm{FeO}$, além do $\mathrm{MgO}$, existe uma solução sólida continua entre os dois óxidos, a fase magnésio-wustita ( $\mathrm{MW}$ ), como mostrado na Figura 2. A temperatura liquidus da MW diminui com o aumento do teor de FeO. Camelli e colaboradores ${ }^{(2)}$, realizaram testes em que um corpo de prova de refratário de $\mathrm{MgO}$ $\mathrm{C}$ foi submerso em escórias com diferentes teores de FeO. Comprovaram que a camada de sólido intermediário formado sobre o refratário é maior para as escórias de mais baixo teor de $\mathrm{FeO}$, como esperado.

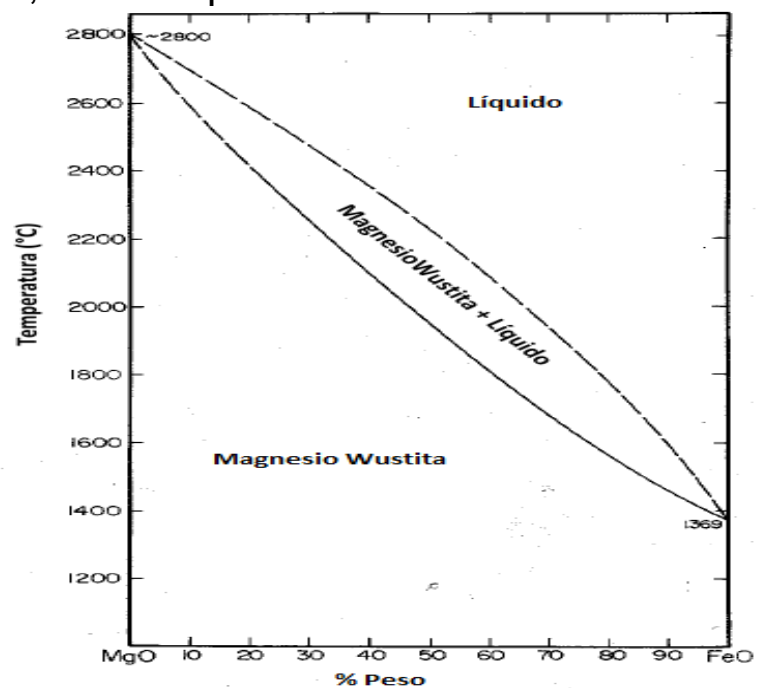

Figura 2- Diagrama MgO-FeO.Adaptado a partir da Ref. [2]

Mills e colaboradores ${ }^{(8)}$ sumarizaram as observações sobre composições empregadas em slag splashing, confirmando a importância do teor de FeO. Propuseram que as misturas pré-tratamento devem conter cerca de $13 \%$ de $\mathrm{FeO}$, para garantir adesão aos refratários sem comprometer a refratariedade, e devem ser supersaturadas em $\mathrm{MgO}$, de modo a evitar a formação de compostos a base de $\mathrm{CaO}$, 
como os silicatos mencionados acima e promover a formação de compostos de $\mathrm{MgO}$ e óxido de ferro, com alto ponto de fusão.

Para a adição de $\mathrm{MgO}$, essencial, portanto, as aciarias normalmente dispõem de cal dolomítica (58\%CaO e $35 \% \mathrm{MgO})$ e dolomita crua $(35 \% \mathrm{CaO}$ e $17 \% \mathrm{MgO})$. $\mathrm{O}$ ideal, do ponto de vista de qualidade da mistura, seria a adição de materiais composto prioritariamente de $\mathrm{MgO}$, contundo está prática nem sempre é viável em função de disponibilidade operacional para a adição, nos sistemas da aciaria. O procedimento de adição de material resinados ricos em MgO e concretos, com custos elevados, podem ser empregados em aciarias em casos de alta criticidade no revestimento refratário. O uso de misturas $\mathrm{MgO}-\mathrm{C}^{(8)}$ também pode ser favorável, assim como tijolos usados desta composição, moídos.

Desta discussão, é evidente que o acerto da composição química da mistura a ser usada no slag splashing ou coating através das adições de cal dolomítica ou dolomita crua é crítico para o sucesso destas operações e é bastante complexo, dependendo da composição e peso da escória de fim de sopro, estado do conversor, temperatura de fim de sopro, etc.

Assim, este trabalho apresenta os resultados do desenvolvimento de um modelo matemático que orienta sobre a quantidade de material para a realização do tratamento do revestimento refratário do conversor pela projeção de escória. Os resultados preliminares do modelo são também apresentados e discutidos.

\section{MATERIAIS E MÉTODOS}

Nesta seção é discutida a formulação do modelo e os dados empregados. Através de cálculos de termodinâmica computacional empregando o programa Thermo-calc e o banco de dados SLAG3 ${ }^{(9)}$ modificado para incluir a magnésio-wustita foi determinada a fração líquida e fases sólidas presentes em equilíbrio, em função da composição e temperatura escórias com composições nas faixas: $\mathrm{MgO}-8$ a 18\%; FeO- 20 a 40\%; CaO - 36 a 57\%; MnO - 2 a 4\% e temperatura na faixa de 1300 a $1725^{\circ} \mathrm{C}$. Com os resultados foi realizada uma regressão linear múltipla que permite o cálculo da fração líquida, como mostrado na Equação 2. Na regressão obteve-se $r^{2}=0.92$ :

Fração Líquida $=a+b x \log \left(\% \mathrm{SiO}_{2}\right)+c x \log (\% \mathrm{FeO})-d x\left(\% M g \mathrm{O}^{0.10}\right)-\left(\right.$ e $x\left(\% \mathrm{CaO} \mathrm{O}^{f}\right)$

Eq.2

$$
\left.+ \text { g } x \ln \left(\text { Temperatura } \text { escória }^{\circ} \mathrm{C}\right)\right)
$$

Empregando o banco de dados citado acima, foi também desenvolvida uma regressão linear múltipla, Equação 3, que permite a estimativa do percentual de magnésio-wustita na escória $\left(\mathrm{r}^{2}=0.92\right)$ :

$\% M W=a+b x$ Temperatura $_{\text {escória }}\left({ }^{\circ} \mathrm{C}\right)+c x \% \mathrm{CaO}+d x \% \mathrm{MgO}+e x \% \mathrm{SiO}_{2}+f x \% \mathrm{FeO}$

No momento que antecede o tratamento do revestimento refratário, a análise química da escória ainda não está disponível. Logo, foi necessário desenvolver um modelo para a estimativa da composição da escória de fim de sopro. Este modelo é baseado em um balanço de massa das principais reações do conversor que influenciam na formação da escória. Usando a composição química e peso do gusa, peso e tipo da sucata, as adições realizadas durante o sopro, oxigênio soprado e composição química ao final de sopro, os coeficientes dos equilíbrios aparentes no fim de sopro foram determinados por regressão. Para este ajuste utilizou-se um banco de dados com mais de 9000 corridas produzidas na CSN.

Também foi necessário desenvolver um modelo que estimasse a temperatura da escória ao final de sopro e após as adições para o tratamento. Para isto estimouse um sobreaquecimento da escória de $50^{\circ} \mathrm{C}$ acima da temperatura da temperatura do aço ao final de sopro. A partir de dados coletados em 50 corridas, estimou-se a 
perda térmica durante o vazamento definindo-se uma constante de perda de temperatura expressa em função do tempo de vazamento $\left({ }^{\circ} \mathrm{C} / \mathrm{min}\right)$. Realizou-se um balanço de entalpia para determinar a variação de temperatura associada a dissolução e calcinação dos materiais adicionados para o tratamento. No caso do tratamento de splashing, adicionalmente foi considerada a perda térmica associada a um sopro de $2000 \mathrm{Nm}^{3}$ de nitrogênio. Assim é possível estimar a temperatura da escória que será empregada no tratamento.

Com a composição estimada para a escória de fim de sopro e a temperatura prevista da escória, o modelo verifica se a fração líquida calculada atende ao requisito chamado "fração líquida ideal". Caso negativo, são definidas as adições necessárias como mostra, de forma sucinta, o fluxograma da Figura 3.

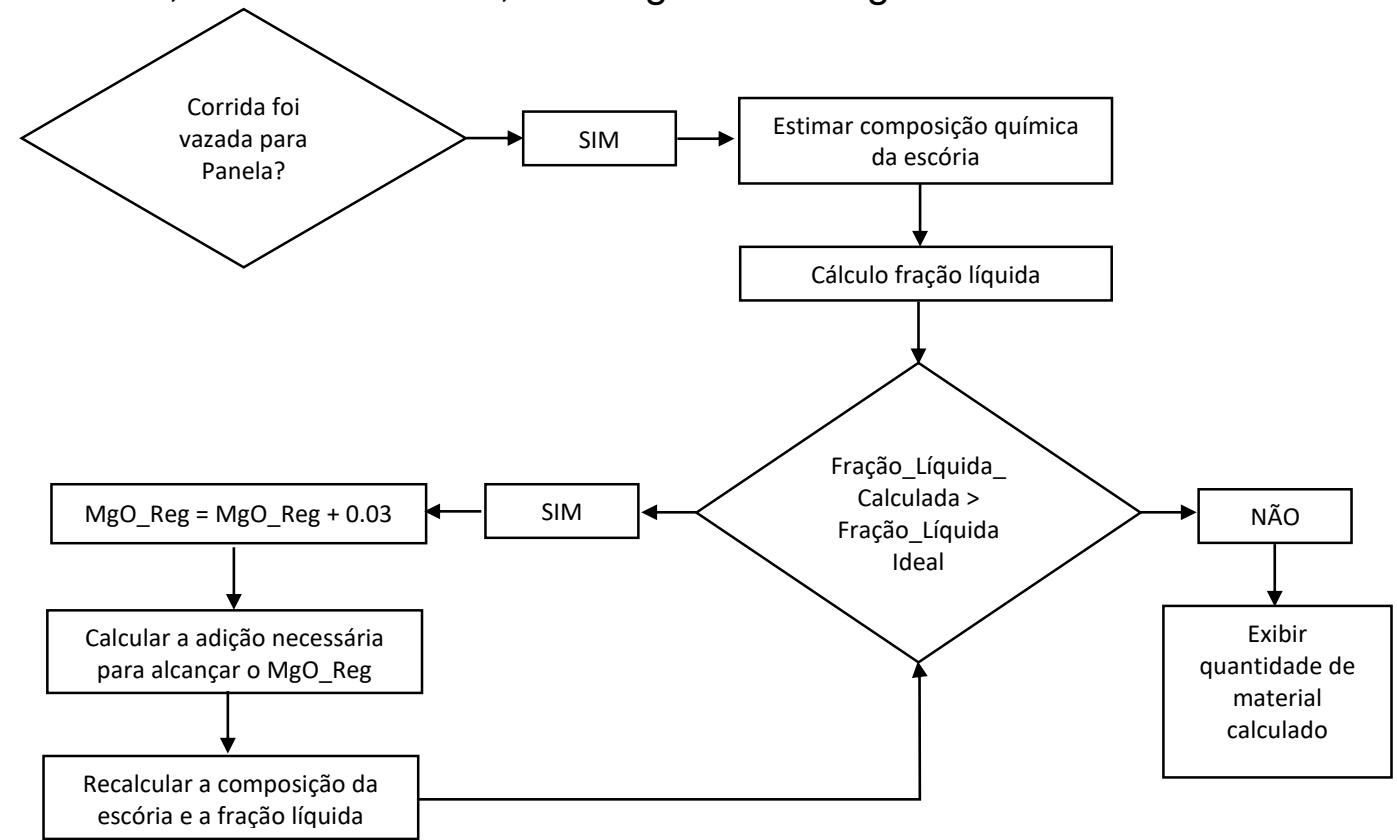

Figura 3- Fluxograma do modelo de adequação da escória para tratamento de proteção do revestimento refratário do conversor

A Figura 4 apresenta a interface do modelo que é usada pelo operador. Como padrão é pré-selecionado o último tipo de tratamento realizado. O usuário seleciona o tipo de material que deseja usar para adição: cal dolomítica, dolomita crua ou os dois simultaneamente. Feito isto, o modelo calcula as adições necessárias para se atingir a fração líquida ideal, estabelecida no modelo. 


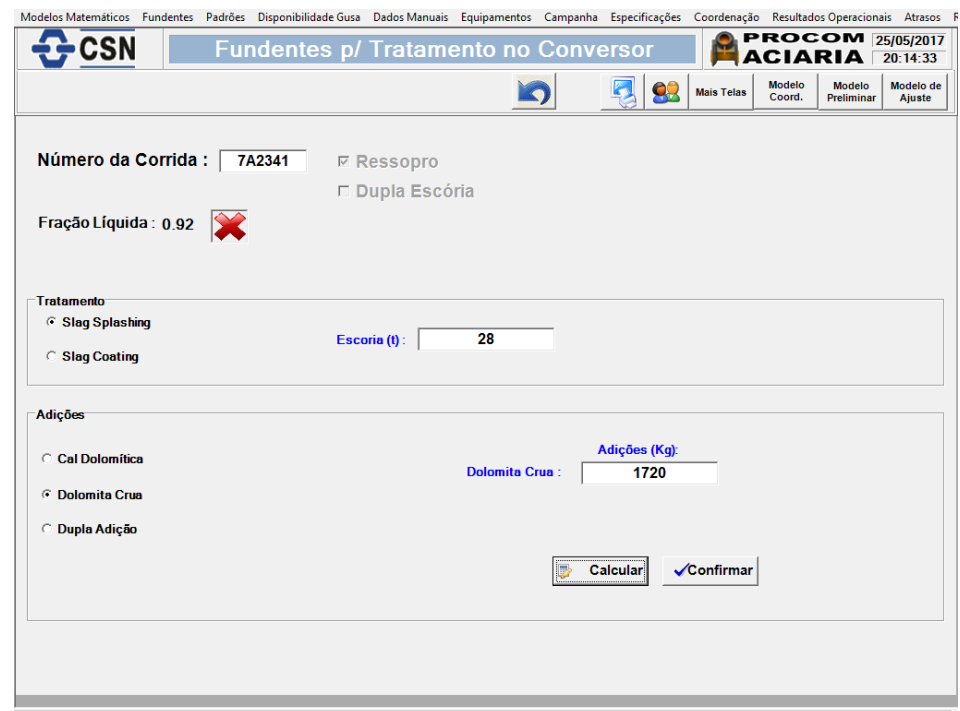

Figura 4- Tela sistema de nível 2 exibida para os operadores de conversores

Os parâmetros do modelo podem ser editados através do sistema da engenharia, como apresentado na Figura 5. Dentre este parâmetro destacam-se a fração líquida ideal, a taxa de queda de temperatura durante o vazamento e o sobreaquecimento da escória. Atualmente, o modelo não avalia a cinética de dissolução do material adicionado. Assim, foram introduzidas variáveis chamadas de "adição extra" que permitem que seja considerado um aumento da adição calculada considerando que nem todo o material está realmente dissolvido na mistura.

\begin{tabular}{|c|c|c|c|c|}
\hline$-\mathrm{CSN}$ & \multicolumn{2}{|c|}{ Parâmetros Slag Coating Splashing } & GACIARIA & \multirow{2}{*}{$\begin{array}{r}\frac{25005 / 2017}{2011: x} \\
\sqrt{7}\end{array}$} \\
\hline & & & & \\
\hline \multicolumn{5}{|c|}{ 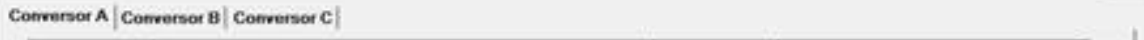 } \\
\hline Dencristo & Descristo & Valee & & \\
\hline AD_CD_COAT & 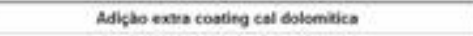 & & & \\
\hline$A D_{2} D_{2} \operatorname{Sen} A$ & 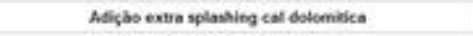 & & & \\
\hline$A D, D C$ COAT & 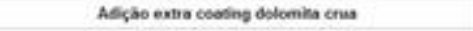 & & & \\
\hline$A D, D$, SPLA & 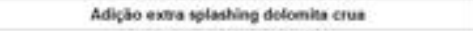 & & & \\
\hline AD_RESF & 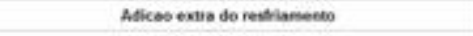 & & & \\
\hline AD S S S & Adicas exve de neblances & & & \\
\hline DOL_CRUA & Sooventa Creso neo Duplas Adichos & & & \\
\hline EMERE_COAT & 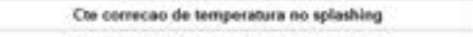 & & & \\
\hline EMER_SPLA & 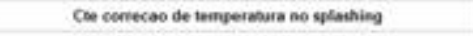 & & & \\
\hline FRAC_UO & Frasho Liquida costiog & & & \\
\hline FAAC UOS & Fracess liquidas Splatiting & & & \\
\hline H.AL__atsF & Peso eborts de redtiamento & & & \\
\hline UAL & Peso alerta da sublances & & & \\
\hline 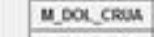 & Pewo maximo de Dolentata cras & & & \\
\hline PCAC De & Perda de Cal Cakabica na Dupla evcoria & & & \\
\hline PDol $x$ & Perde de Cal Doloberitica na Ouplas ececoria & & & \\
\hline PEso Ese & Peno Focoria Costim & & & \\
\hline Quena 12 & 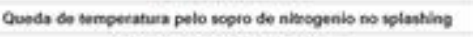 & & & \\
\hline SOBAE_ESC & 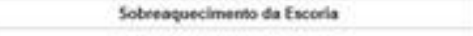 & & & \\
\hline TAXA TEWP & 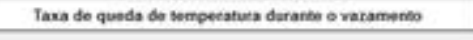 & & & \\
\hline
\end{tabular}

Figura 5- Tela sistema nível 2 disponível para a engenharia.

\section{RESULTADOS E DISCUSSÃO}

\subsection{Validação dos cálculos das adições a partir do modelo de escória de fim de sopro}

Para permitir o cálculo das adições antes de se dispor da análise química da escória de fim de sopro, como discutido no item 2, acima, foi desenvolvido um modelo de previsão da composição da escória de fim de sopro. 
Formulou-se um modelo de balanço de massa, empregando regressões múltiplas, para permitir o cálculo aproximado da composição química da escória de fim de sopro. Para avaliar a qualidade deste modelo, do ponto de vista de splashing e coating, foram comparadas as frações líquidas calculadas usando (a) a composição da escória prevista pelo modelo e (b) a composição analisada da escória de fim de sopro. Na Figura 6 a fração líquida da escória, calculada pelos dois métodos, é comparada.

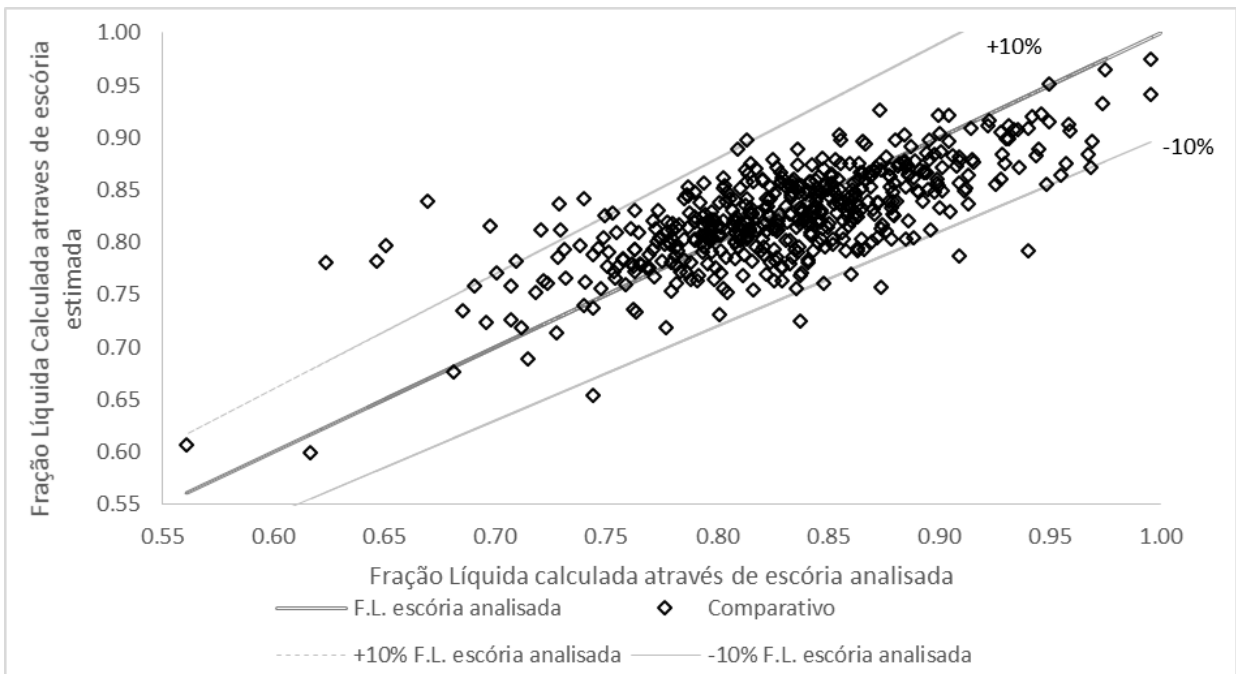

Figura 6-Gráfico comparativo da fração líquida calculada empregando a composição da escória calculada pelo modelo formulado neste trabalho e a mesma fração calculada empregando a composição da escória de fim de sopro analisada por espectrômetro de emissão ótica de Raios- $X$.

$\mathrm{Na}$ Tabela 1 apresenta-se o resumo dos mesmos resultados para 500 corridas. Observa-se que a estimativa de fração líquida empregando a composição e peso da escória estimada para o fim de sopro é muito boa, quando comparada com o cálculo realizado com a escória analisada, posteriormente.

Tabela 1- Média e Desvio Padrão do Grupo fração líquida calculada através da escória gerada por regressões e o Grupo fração líquida calculada através da escória analisada via espectrômetro de emissão ótica de Raios- $X$

\begin{tabular}{|c|c|c|}
\hline & $\begin{array}{c}\text { Fração Líquida (Escória Estimada } \\
\text { pelo modelo) }\end{array}$ & Fração Líquida (Escória Analisada) \\
\hline Média & 0.83 & 0.83 \\
\hline Desvio Médio & 0.04 & 0.05 \\
\hline
\end{tabular}

Da mesma forma, calculou-se o peso de material a adicionar, usando o modelo de splashing estabelecido neste trabalho, empregando-se a composição estimada e a composição real da escória de fim de sopro. A Figura 7 mostra as diferenças nas adições previstas. A diferença média observada em 10 corridas escolhidas ao acaso foi de $114.3 \mathrm{~kg}$. A diferença máxima, nestas corridas, foi de cerca de $300 \mathrm{~kg}$. Considerando que as adições calculadas se situam tipicamente entre 1000 e $2000 \mathrm{~kg}$, o desvio é consideravelmente pequeno e aceitável. 


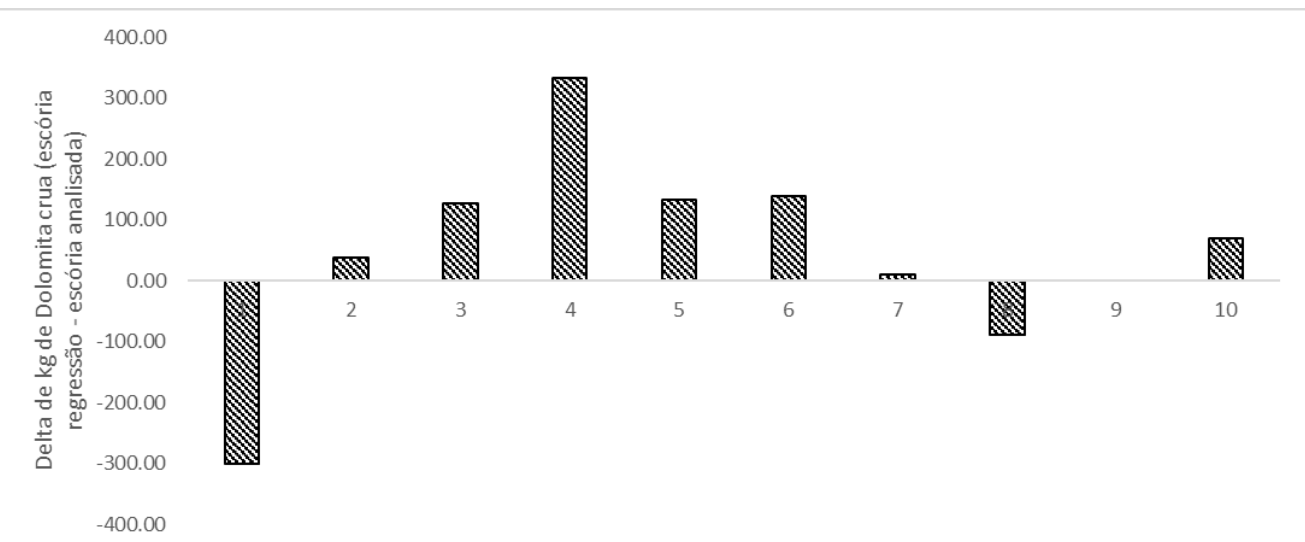

Figura 7 - Comparativo entre a adição calculada pelo modelo usando a composição da escória prevista pelo modelo de escória ou a composição obtida, posteriormente, por análise química da escória de fim de sopro.

\subsection{Validação do modelo de temperatura}

Para a validação do modelo de temperatura, utilizou-se uma câmera termográfica que permite medições de temperatura em pontos definidos do conversor. Assim, foram medidas temperaturas da escória presente no conversor e comparadas com os valores estimados. A Figura 8 permite concluir que a diferença entre o valor calculado pela estimativa de temperatura se assemelha de modo satisfatório da temperatura medida.

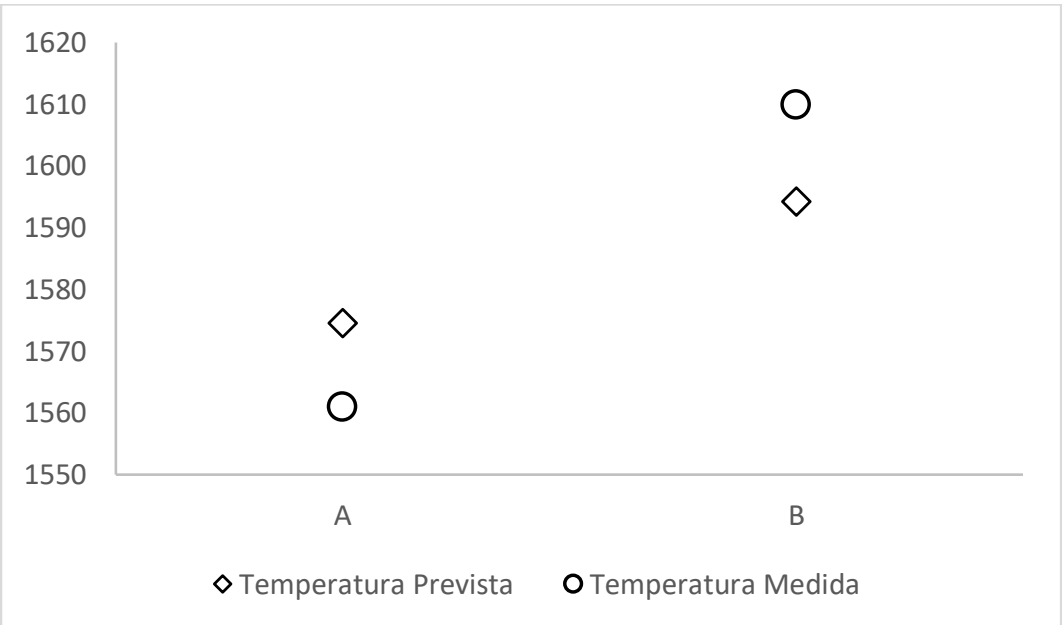

Figura 8- Comparativo da temperatura prevista pelo modelo e temperatura medida via câmera termográfica.

\subsection{Eficiência do modelo de adequação da escória para tratamento de proteção do revestimento refratário do conversor}

Para verificar a eficiência da adição sugerida pelo modelo, foram amostradas, como dados preliminares, 6 corridas produzidas em um conversor. A amostragem foi feita ao final de sopro e após o splashing. As amostras foram obtidas através da imersão de um vergalhão e da solidificação da escória em sua superfície. A escória solidificada foi removida do vergalhão após resfriamento ao ar.

Estimada a composição química e a massa da escória ao final de sopro, a composição química da escória após o splashing e o peso e a composição químico padrão do material adicionado para o tratamento, foi possível realizar um balanço de massa e determinar a massa e a composição da escória aderida ao refratário. Para este cálculo foi considerada a premissa de total dissolução do material na mistura, o que pode não ter ocorrido e está sendo investigado em outro projeto. 
A Tabela 2 apresenta resultados obtidos no tratamento das corridas amostradas. É importante lembrar que o volume de nitrogênio soprado depende diretamente da avaliação visual do operador em relação ao splashing produzido. Quando o splashing começa a perder intensidade, ou seja, a escória tem mais dificuldade de ser espalhada devido a sua elevada viscosidade, o sopro é encerrado e a escória restante é removida do conversor.

Tabela 2- Dados referente ao tratamento de slag splashing das corridas amostradas

\begin{tabular}{|c|c|c|c|c|}
\hline CORRIDA & DOLOMITA CRUA & CAL DOLOMÍTICA & VOLUME N2 & $\begin{array}{c}\text { FRAÇÃO LíQUIDA } \\
\text { FINAL SOPRO }\end{array}$ \\
\hline A & 0 & 0 & 1205 & 0.82 \\
\hline B & 0 & 389 & 1331 & 0.83 \\
\hline C & 865 & 0 & 1064 & 0.81 \\
\hline D & 281 & 0 & 1144 & 0.80 \\
\hline E & 0 & 0 & 1082 & 0.78 \\
\hline F & 0 & 0 & 1074 & 0.86 \\
\hline
\end{tabular}

Analisando a Figura 9 verifica-se que, como desejado, a escória aderida ao refratário contém mais $\mathrm{MgO}$ que a escória de final de sopro e também que a escória não aderida._Este fato era esperado devido à adição de materiais contendo $\mathrm{MgO}$ à escória e ao sopro de nitrogênio que proporciona a mistura destes materiais com a escória. A escória $A$, por sua vez, chama a atenção por apresentar um pequeno aumento de $\mathrm{MgO}$ embora não tenha sido feita adição contendo $\mathrm{MgO}$ na preparação para o tratamento. Ainda assim, como esperado, o teor de $\mathrm{MgO}$ da escória não aderida foi inferior ao da escória de final de sopro.

Conforme comentado, embora o ideal, para o processo, seria realizar adições ricas em $\mathrm{MgO}$, as condições operacionais e estratégias levam a CSN trabalhar com cal dolomítica e dolomita crua como adições para splashing ou coating. Como estes materiais apresentam apreciável teor de $\mathrm{CaO}$, verificou-se aumento considerável de $\mathrm{CaO}$ nas escórias após o tratamento em relação a escória final de sopro. 


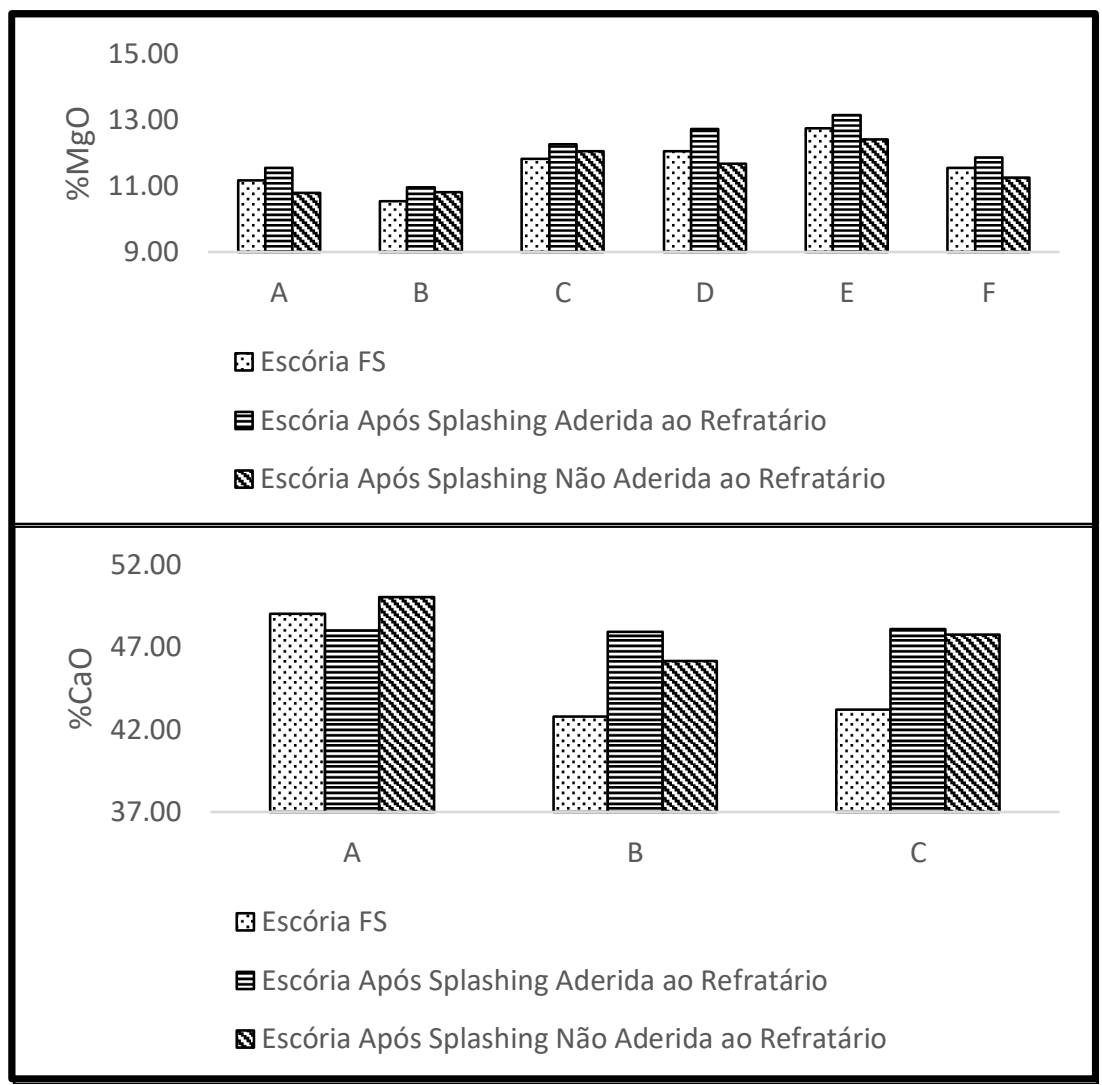

Figura 9 - \%MgO \% \%aO da escória das corridas amostradas após o final de sopro e após o splashing (a amostra escória após o splashing não aderida ao refratário foi analisada e a escória após splashing não aderida ao refratário foi calculada).

Em todas as corridas foi observado a redução da fração líquida da escória aderida ao refratário em relação a escória de final de sopro (Figura 10). Em média, a fração líquida após tratamento ficou em 0,70 , valor muito próximo do objetivado pelo modelo de engenharia da CSN.No caso em que não foi adicionado qualquer material a escória de final de sopro (corrida A), a queda da fração líquida se deve, exclusivamente, a perda térmica da escória.

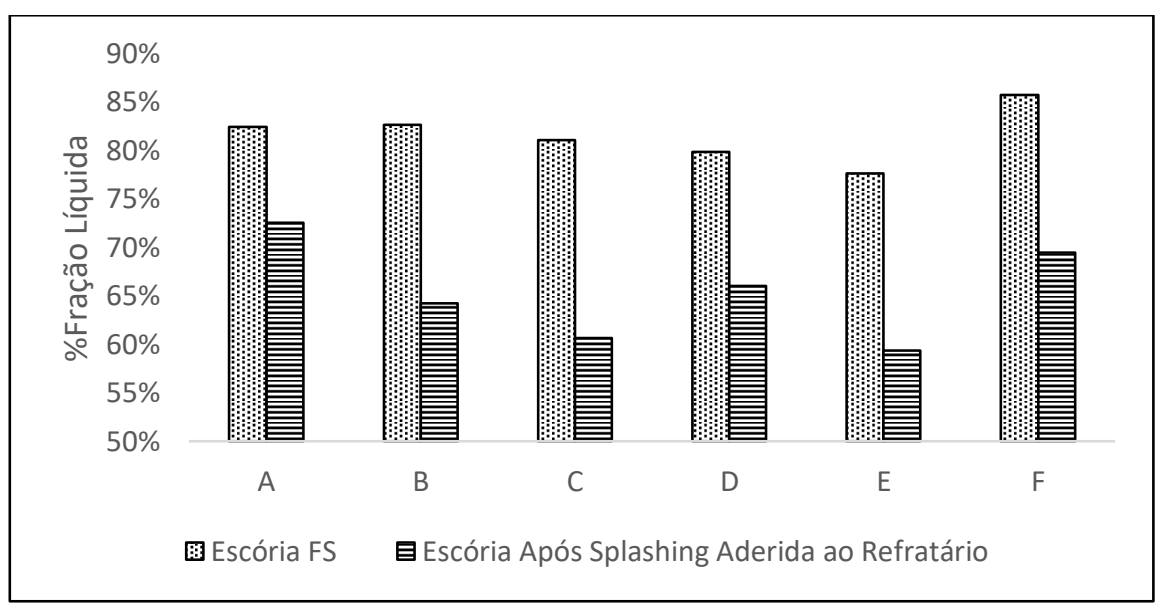

Figura 10 - Fração líquida calculada através da Equação I para a escória final de sopro e escória após splashing Aderida ao Refratário.

Utilizando-se a Equação 3, calculou-se o percentual da fase magnésio- wustita presente na escória (Figura 11). Em todos os casos houve aumento da fração desta 
fase, como desejado para um tratamento eficiente para a proteção do revestimento refratário.

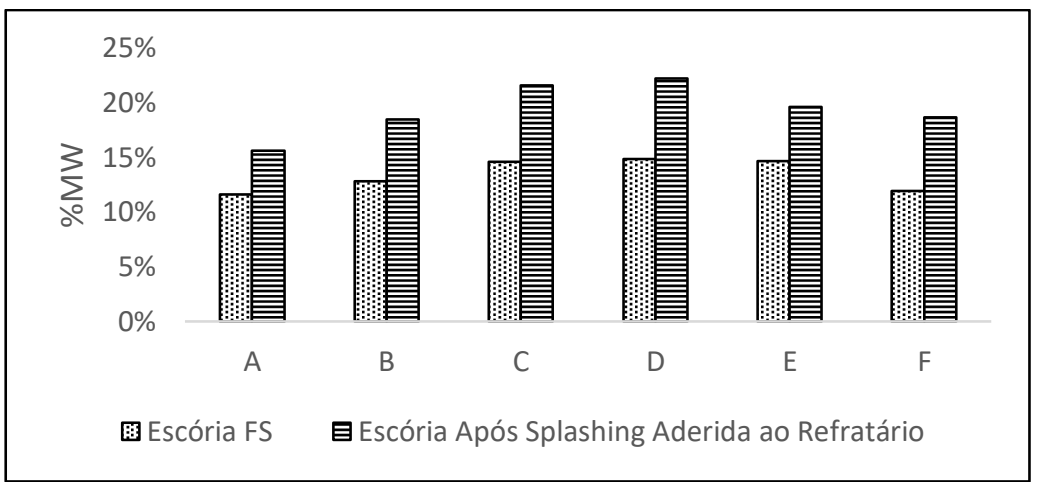

Figura 11- Percentual da fase Magnesio-wustita na escória ao final de sopro e na escória aderida ao revestimento refratário após o splashing.

Utilizando a Equação 1, estimou-se a temperatura liquidus da escória ao final de sopro e da escória aderida ao revestimento refratário após o splashing. A Figura 12 apresenta a diferença entre estas duas temperaturas. Nas corridas B e C houve expressivo aumento da temperatura liquidus da escória. Na corrida, A, sem adições, o aumento não foi expressivo.

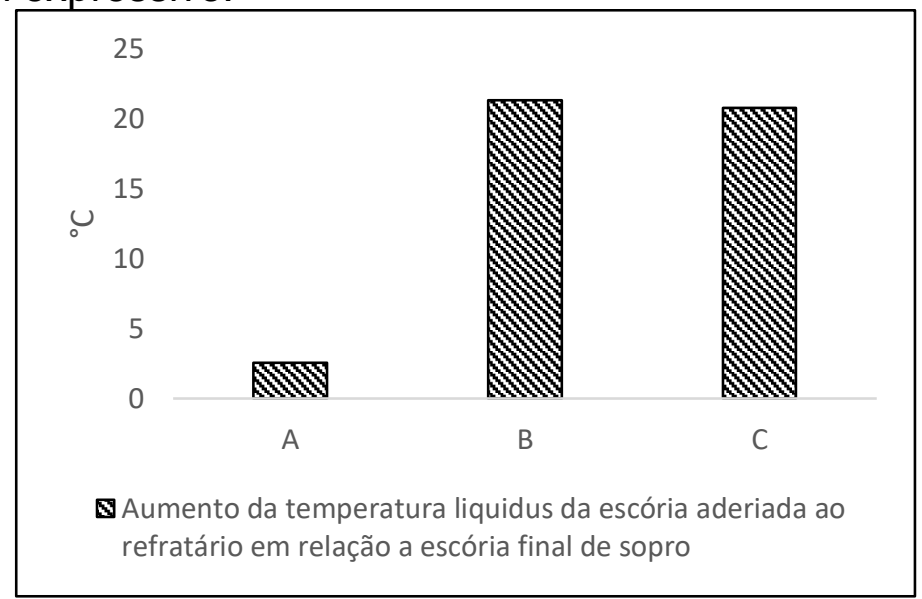

Figura 12- Delta entre a temperatura liquidus da escória aderida ao revestimento refratário e da escória de final de sopro.

\section{CONCLUSÃO}

Dentre as diversas variáveis importantes para o tratamento adequado do revestimento refratário do conversor foram escolhidas duas variáveis fundamentais: a fração líquida da escória e a fase sólida a ser formada. O modelo formulado, com base nos dados da corrida (composição química e massa do gusa, tipo e massa de sucata, adições de fundentes, refrigerantes e exotérmicos, volume de sopro de oxigênio e composição química do aço) alimenta regressões lineares múltiplas, que possibilitam a previsão da composição química da escória. Com os dados gerados, é possível o cálculo da fração líquida da escória ao final de sopro. Os resultados mostram que esta previsão é bastante satisfatória.

A partir da fração líquida da escória ao final de sopro e a fração líquida ideal visada, o modelo calcula a perda térmica da escória durante o vazamento e prevê a perda que ocorrerá durante o tratamento. O modelo sugere então, ao operador, a adição a ser feita para se alcançar a fração líquida e a fase desejada. Os resultados dos testes indicam que o modelo produz resultados em ótimo acordo com as metas fixadas, não apenas em fração líquida, mas também na quantidade da fase magnésiowustita. 
Apesar dos tratamentos de slag splashing e slag coating serem rotinas tradicionais em diversas Aciarias, inclusive na CSN, classicamente estes procedimentos eram tratados como totalmente dependentes da avaliação do operador. O modelo desenvolvido neste trabalho estabelece um método tecnológico e científico que permite orientar esta operação, com excelentes resultados, reduzindo o caráter empírico do tratamento.

\section{Agradecimentos}

Agradecimento a Universidade Federal Fluminense, a Companhia Siderúrgica Nacional, a Magnesita e Saint-Gobain.

\section{REFERÊNCIAS}

1 Fruehan R, editor. Making, Shaping, and Treating of Steel, Steelmaking and Refining Volume. 11th Edition. Pittsburgh PA: AISE Steel Foundation; 1998.

2 Camelli S, Brandaleze E, Madias J, Topolevsky R. PROTECTION OF CONVERTER LINING WITH SLAG ADHERENCE MECHANISMS. In: XXXIII Seminário de Fusão, Refino e Solidificação dos Metais [Internet]. ABM; 2001

3 A Costa e Silva. Estimating viscosities in iron and steelmaking slags in the $\mathrm{CaO}$ Al2O3-MgO-SiO2-(TiO2) system with basis on a thermodynamic model. Journal of Materials Research and Technology. 2012;1:154-60.

4 Ito K, Fruehan RJ. Study of the foaming of CaO-SiO2-FeO-Slags Part II. Dimensional analysis and foaming in Iron and Steelmaking processes. Metallurgical Transactions B. 1989;20B(August):515-21.

5 Barron MA, Medina DY, Hilerio I. CFD Analysis of Influence of Slag Viscosity on the Splashing Process in an Oxygen Steelmaking Converter. Modeling and Numerical Simulation of Material Science. 2013;03(03):90-3.

6 J.C.Grosjean, P.V. Riboud . Consistance das laitiers de convertisseurs et tartinage. Revue de Métallurgie. Jullet 1983, 571-584.

7 Slag Atlas - Verein Deutscher Eisenhüttenleute, prepared by the Committee for Fundamental Metallurgy. 1981

8 K C Mills, Y Su, A B Fox, Z Li, R P Thackray, H T Tsai. A review of slag splashing. ISIJ International. 2005;45(5):619-33.

9 Andersson JO, Helander T, Höglund L, Shi P, Sundman B. Thermo-Calc \& DICTRA, computational tools for materials science. Calphad. 2002;26(2):273312.

10 CHRISÓSTOMO, W. B. ; PEREIRA, C. L. ; COSTA E SILVA, A. . AVALIAÇÃO DA SOLUBILIDADE DE MgO EM ESCÓRIAS DE ACIARIA ATRAVÉS DE DIFERENTES MÉTODOS DE CÁLCULO. In: XXX Seminário sobre Fusao, Refino e Solidificacao de Acos, Belo Horizonte, MG, 1999, Belo Horizonte, MG. São Paulo: ABM, 1999. 